\title{
Large-Scale Read/Write Margin Measurement in 45nm CMOS SRAM Arrays
}

Zheng Guo, Andrew Carlson, Liang-Teck Pang, Kenneth Duong, Tsu-Jae King Liu, Borivoje Nikolic

Department of Electrical Engineering and Computer Sciences, University of California, Berkeley, CA 94720, USA

*Phone: (510) 643-2558, Fax: (510) 643-2636, E-mail: zhengguo@eecs.berkeley.edu

\begin{abstract}
Distributions of read and write noise margins in large CMOS SRAM arrays are investigated by directly measuring the bit-line current during bitline / wordline (write) or cell supply (read) voltage sweep in a $768 \mathrm{~Kb} 45 \mathrm{~nm}$ CMOS SRAM test-chip. Good correlation between write/read margin estimates through the bit-line measurements and the DC read SNM (RSNM) and $\mathrm{I}_{\mathrm{W}}$ measurements in small on-chip SRAM macros with wired-out storage nodes are demonstrated. Four common writeability metrics are correlated and compared. Array-level characterization of SRAM cell read stability and writeability allow fast and accurate characterization of highdensity SRAM arrays is scalable for capturing up to 6 standard deviations of parameter variations.
\end{abstract}

\section{Introduction}

Continued increase in the process variability is perceived to be a major challenge in future technology scaling. To satisfy the functionality of hundreds of millions of SRAM cells in current ondie cache memories, the design has to provide more than 6 standard deviations of margin to parameter variations. Margins have been estimated through TCAD simulations or by measuring DC $\mathrm{read} /$ write margins in small SRAM macros with wired-out storage nodes [1-2] and direct cell current measurement in large SRAM arrays [3]. In this work, SRAM cell read stability and writeability margins are estimated through bitline currents and compared to direct DC RSNM and $\mathrm{I}_{\mathrm{W}}$ measurements.

\section{Measurement Methodology}

Writeability: During a write operation, a low-going $\mathrm{BL}_{\mathrm{R}}$ voltage pulls down on the ' 1 ' storage node as the WL is held high until the trip point of the inverter formed by PR and NR is reached, and a bit cell is successfully written (Fig. 1a). In this work, the measurements of four commonly used writeability metrics are compared and correlated. The BL write margin (BLWM) is the maximum BL voltage able to flip the cell state and can be measured through monitoring the $\mathrm{BL}_{\mathrm{L}}$ current at the ' 0 ' storage ( $\mathrm{I}_{\mathrm{MEAS} 1}$ in Fig. 1a) while ramping down the $\mathrm{BL}_{\mathrm{R}}$ voltage with $\mathrm{WL}$ held high. The voltage at $\mathrm{BL}_{\mathrm{R}}$ that induces a sudden drop in $\mathrm{I}_{\text {MEASI }}$ is the write margin of the cell (Fig. 1b). Similarly, a WL write margin (WLWM) can be defined as the maximum of $\left(\mathrm{V}_{\mathrm{DD}}-\mathrm{V}_{\mathrm{WL}}\right)$ in a WL voltage sweep (Fig. 1c) while monitoring the change in the BL current at the ' 1 ' storage node ( $\mathrm{I}_{\text {MEAS2 }}$ in Fig. 1a). Write margin can also be measured as the writeability current $\left(\mathrm{I}_{\mathrm{W}}\right.$, as defined in Fig. $4 \mathrm{c}$ and [4]), or the write noise margin derived from the butterfly curves (BFC) [1].

Read Stability: The read stability of an SRAM cell is conventionally quantified by the cell read static noise margin (RSNM) [5] and is highly sensitive to the cell supply. To characterize the read stability of an SRAM cell in a large array, BL current at the ' 0 ' storage node $\left(\mathrm{I}_{\mathrm{MEAS} 1}\right)$ is monitored while ramping down the cell supply with the bit-lines pre-charged and WL driven by the nominal supply. The difference between the nominal supply and the cell supply causing $\mathrm{I}_{\text {MEASI }}$ to drop gives the read margin (RM) of the SRAM cell under test (Fig. 1d).

Array Implementation: A $2 \mathrm{~mm} \times 2 \mathrm{~mm}$ test chip (Fig. 2) is implemented in a low-power 7M1P 45nm CMOS process, consisting of eight $32 \mathrm{~kb}$ and eight $64 \mathrm{~kb}$ SRAM arrays as well as eighteen 40x20 SRAM macros, where 20 cells in each macro have all internal nodes externally accessible. The high-speed SRAM cell under study has an area of $0.374 \mathrm{um}^{2}$. Each large array consists of 256 columns and either $128(32 \mathrm{~Kb})$ or $256(64 \mathrm{~Kb})$ rows. Bitline currents are measured through wide, long channel, thick oxide CMOS pass-gates driven by a separate supply to suppress leakage in unaccessed bitlines (Fig. 3).

\section{Results}

Write/Read Margin Metric Comparison: $\mathrm{I}_{\mathrm{W}}, \mathrm{WL}$, and BFC WMs were measured for several hundred cells with externally accessible storage nodes in SRAM macros. WL and BL WMs were measured for hundreds of thousands of cells in the large SRAM arrays. Fig. 5a shows the WM distribution densities measured using the four different metrics. BLWM has a higher mean-to-standard-deviation ratio $(\mu / \sigma)$ than $I_{W}$ and BFC. WLWM has a tighter distribution than BLWM and the highest $\mu / \sigma$, due to the fact that the WL sweep modulates access transistor strength on both sides of the cell, thereby assisting the write operation. The $\mathrm{I}_{\mathrm{W}} \mathrm{WM}$ has higher $\sigma$ due to a heightened sensitivity to STI-related edge effects on strain [6] in the smaller macros (Fig. 6). WMs measured through BL, WL sweep, and by $\mathrm{I}_{\mathrm{W}}$ show excellent correlations (Fig. 5b, c); suggesting that BLWM and WLWM are valid for large-scale write margin measurements where measurements for $\mathrm{I}_{\mathrm{W}}$ and $\mathrm{BFC}$ are not feasible. The BFC WM does not correlate well to the other write margins, due to the high write-ability of the studied cell.

Fig. 7 shows measured RM distribution densities using both RSNM and $\mathrm{V}_{\text {CELL }}$ sweep. The distributions show excellent agreement in shape and $\mu / \sigma$. A strong correlation between the two metrics is also found through Monte Carlo simulations (Fig. 7b).

Array Measurement Results: Measured BLWM distributions and measured $\mathrm{V}_{\mathrm{CELL}}$ sweep RM distributions from several different arrays across the chip are plotted in Fig. 8 and Fig. 9. Measurements reveal up to $6 \%$ shift in the mean of measured BLWM depending on the value stored. The shift is consistent throughout the chip, suggesting a systematic processing effect. The shift in measured RM distribution is negligible. Fig. 10a shows the layout of a 4 cell cluster in the array and Fig. 10b plots normalized mean of both measurements for each orientation in the 4 cell cluster. Mismatch between the two sides of the SRAM cell is observed in the RM and in BLWM measurements. The results suggest that cells mirrored along the direction of the poly show positive correlation in cell margins (e.g. orientation $\mathrm{A}$ and $\mathrm{C}$ ), where as cells mirrored across poly show negative correlation in cell margins (e.g. orientation A and B).

\section{Conclusion}

A measurement methodology to characterize SRAM cell read stability and writeability in large SRAM arrays has been introduced and demonstrated for the first time in the $45 \mathrm{~nm}$ technology node. Measurements show good correlation to DC RSNM and $I_{W}$ measurements conducted in small SRAM macros with wired-out storage nodes. Mean to standard deviation yield estimates were shown to depend on the writeability metric used. The ability to characterize stability in large SRAM arrays allows much more statistics to be captured and is critical for the investigation of future SRAM scalability. This methodology can be extended to capture up to 6 standard deviations of parameter variations by increasing the SRAM array size.

\section{Acknowledgement}

The authors acknowledge the NSF RI Grant No. 0403427, chip fabrication donation of STMicroelectronics, and the support of the Center for Circuit \& System Solutions (C2S2) Focus Center.

\section{References}

[1]A. Bhavnagarwala et al., IEDM 2005.

[2]A. Bhavnagarwala et al, VLSI 2007.

[3]C.H. Hsiao et al, MTDT 2005.

[4]C.Wann et al, VLSI-TSA 2005.

[5]E. Seevinck et al. IEEE JSSC 1987.

[6] S.E. Thompson, et al, IEEE EDL, 2004. 


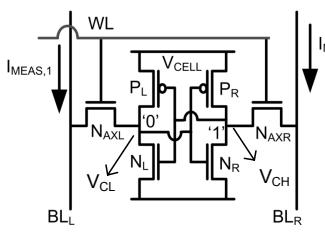

(a)

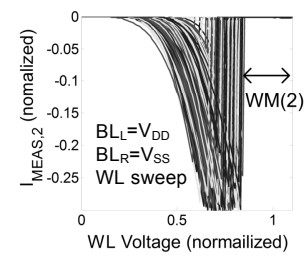

(c)

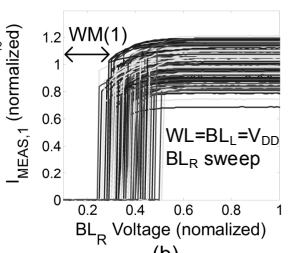

(b)

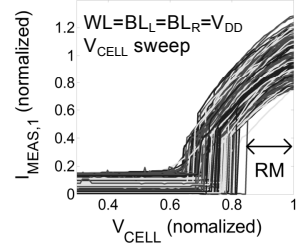

(d)
Fig.1: Schematic (a) for read/write margin measurement setup, and measured waveforms for write margin using BL sweep (b) WL sweep (c) and read margin using $\mathrm{V}_{\mathrm{CELL}}$ sweep (d)

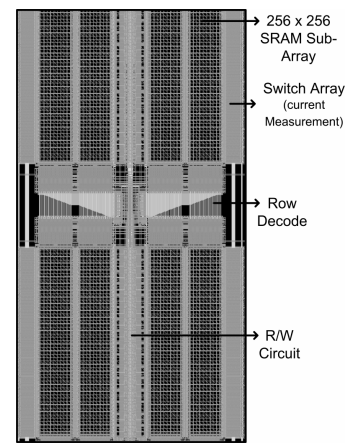

Fig. 3: (left) a 4x256x256 SRAM array with addressable switch array for bit-line current measurement

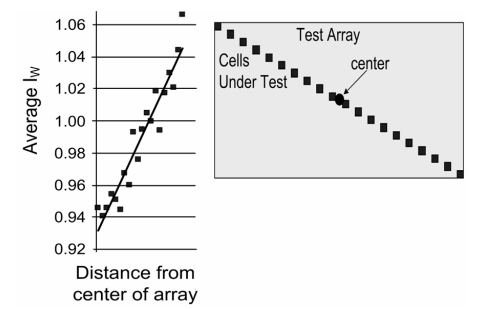

Fig. 6: Average $I_{W}$ as a function of distance from center of array showing high sensitivity to STIrelated edge effects in the smaller macros

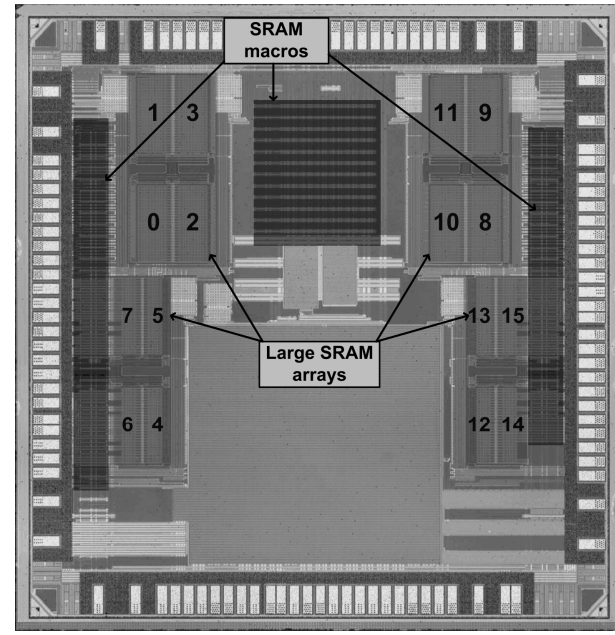

Fig. 2: Die photo of the $45 \mathrm{~nm}$ CMOS test-chip

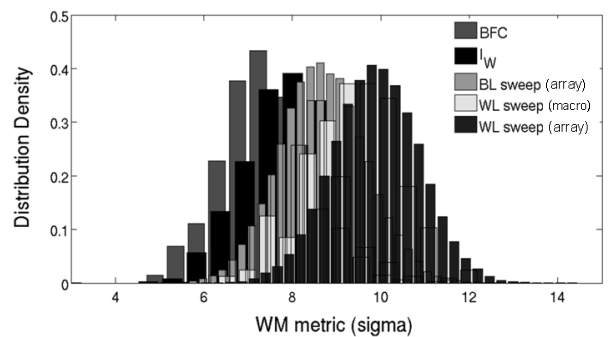

(a)

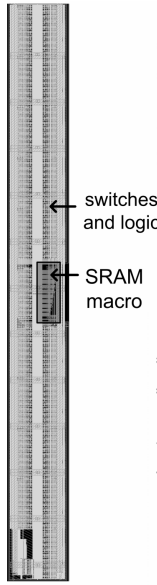

(a)

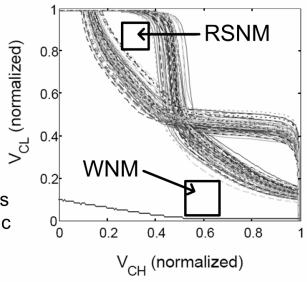

(b)

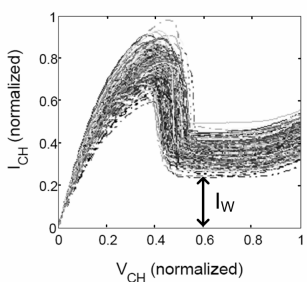

(c)

Fig. 4: (a) a 40x20-cell macro consists of 20 cells that have externally accessible internal nodes for Read $\mathrm{SNM}, \mathrm{BFC}$ WNM (b) and $\mathrm{I}_{\mathrm{W}}$ (c) measurement

Fig. 5: Measured write margin distributions using 4 different metrics (a) with 3 metrics BFC, $I_{W}$, WL sweep - measurable in SRAM macros and 2 metrics - WL sweep, BL sweep directly measurable from large SRAM arrays. Good correlation is established from measurements between $\mathrm{I}_{\mathrm{W}}, \mathrm{WLWM}$, and $\operatorname{BLWM}(\mathrm{b}, \mathrm{c})$

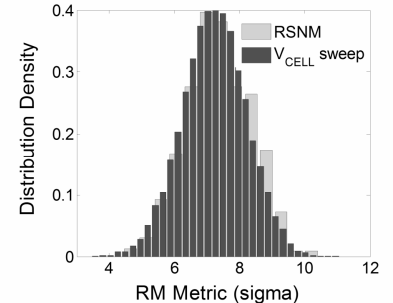

(a)

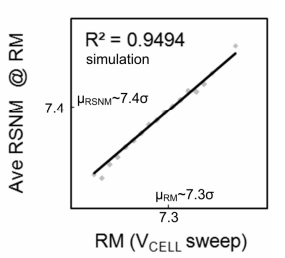

(b)
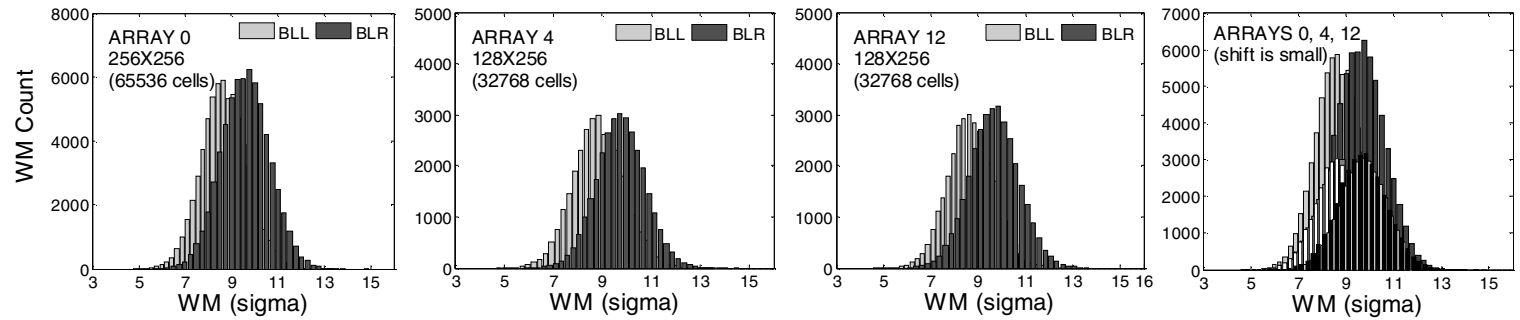

Fig. 8: Write margin (using BL sweep) distributions for 3 different sub-arrays showing up to $6 \%$ shift in the mean between the left and right halves of an SRAM cell (determined by either a $\mathrm{BL}_{\mathrm{L}}$ sweep or a $\mathrm{BL}_{\mathrm{R}}$ sweep; left 3 plots above) and marginal shift in the mean between arrays at different locations across the die (right).
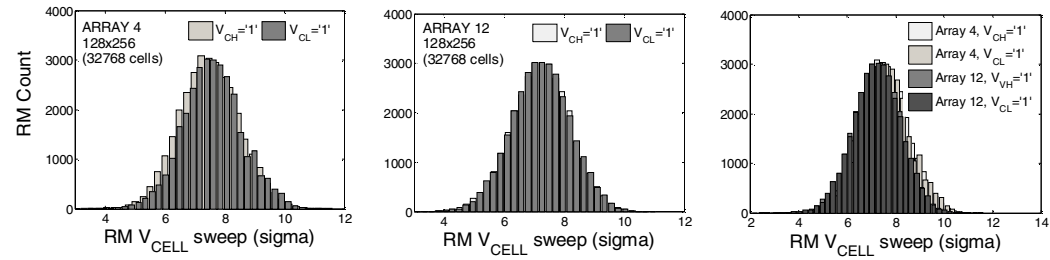

Fig. 9: Read margin (using $V_{\text {CELL }}$ sweep) distributions for 2 sub-arrays showing no systematic shift in mean between the left and right halves of an SRAM cell (left 2) and marginal shift in the mean between arrays at different locations (right)
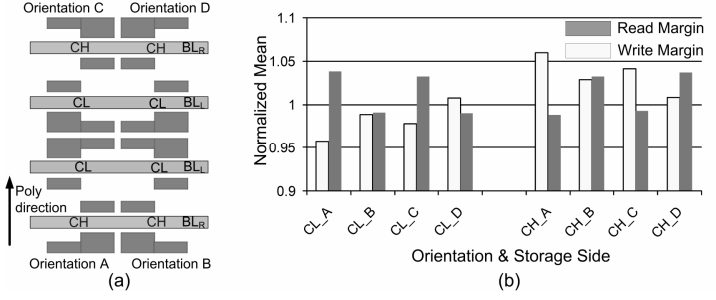

Fig. 10: (a) layout of a 4 cell cluster in the SRAM array. (b) normalized mean of RM and WM for each layout orientation. 